\title{
ASPECTOS DA ECOlOgia RePRodUtiva DE Pera glabrata (Schott) Poepp. ex Baill. (EUPHORBIACEAE) EM UMA ÁREA DE CERRADO NO ESTADO DE SÃO PAULO ${ }^{1}$
}

Juliana Ribeirão de Freitas², Rodolfo Antônio de Figueiredo ${ }^{3}$, Igor Chiosini de Nadai ${ }^{4}$ e Lucas Hardman ${ }^{4}$

RESUMO - Pera glabrata é uma árvore que apresenta ampla distribuição no Brasil. A espécie vegetal é de importância para a conservação e recuperação de áreas degradadas, pois está presente em áreas impactadas, produz e dispersa grande quantidade de sementes e constitui-se em fonte alimentar para elevado número de espécies animais. Apesar da importância fitossociológica da espécie, ainda não existem estudos que abordem a sua ecologia reprodutiva. Este trabalho teve como objetivo caracterizar aspectos da fenologia reprodutiva, da morfologia floral, dos sistemas reprodutivo e de polinização e da dispersão de sementes da espécie. O estudo foi realizado em uma área de Cerrado no município de São Carlos, SP. Verificou-se que Pera glabrata é dioica e apresenta floração massiva e as flores dos dois sexos são pequenas, involucradas, amarelas e de antese diurna. As flores masculinas apresentam néctar e emitem odor adocicado, e as femininas não oferecem recursos perceptíveis aos visitantes florais. As flores foram visitadas por 32 espécies de Diptera e Hymenoptera de pequeno porte. Ocorre a formação de frutos e sementes por autogamia. Foram identificadas 25 espécies de aves visitando indivíduos com frutos maduros, das quais 16 ingeriram as sementes ariladas. Pera glabrata é autogâmica, com síndrome de polinização por diversos pequenos insetos e com dispersão ornitocórica de suas sementes.

Palavras-chave: Reprodução, Polinização e Dispersão.

\section{ASPECTS OF PERA GLABRATA (Schott) Poepp. ex Baill. (EUPHORBIACEAE) REPRODUTICTIVE ECOLOGY IN A CERRADO AREA IN SÃO PAULO STATE}

\begin{abstract}
Pera glabrata is a widely distributed tree in Brazil. The plant species is important for conservation and restoration of degraded areas due its presence in impacted environments. Besides, the production and the dispersion of large quantities of seeds, provide food supply for many species of animals. Despite its phytosociological importance, there are no studies addressing its reproductive ecology. The objectives of this study are to characterize the reproductive phenology, floral morphology, breeding system and pollination and seed dispersal of the species. The study was conducted in an area of cerrado in São Carlos (SP). It was found that Pera glabrata is dioecious, showing massive flowering and the flowers of both sexes are small, involuted, yellow and with diurnal anthesis. The male flowers provide nectar, and emit a sweet odor, while the female ones do not offer visible reward to visitors. The flowers were visited by 32 small species of Diptera and Hymenoptera. Fruits and seeds were developed through autogamy. Twenty-five bird species visited individuals bearing ripe fruits, and 16 species ingested arilate seeds. In conclusion, Pera glabrata is an autogamous species, with several small insects pollination system and ornithocoric dispersal of its seeds.
\end{abstract}

Keywords: Reproduction, Pollination and Seed dispersal..

\footnotetext{
${ }^{1}$ Recebido em 17.06.2010 e aceito para publicação em 31.10.2011.

${ }^{2}$ Programa de Pós-Graduação em Ecologia e Recursos Naturais pela Universidade Federal de São Carlos, UFSCAR, Brasil. Email: <juzinhabio@gmail.com>

${ }^{3}$ Departamento de Agroecologia da Universidade Federal de São Carlos, UFSCAR, Brasil. E-mail: <raf@cca.ufscar.br>

${ }^{4}$ Graduação em Ciências Biológicas pela Universidade Federal de São Carlos, UFSCAR, Brasil. E-mails: <igordenadai@hotmail.com>

<lucashardman@hotmail.com>
} 


\section{INTRODUÇÃO}

A família Euphorbiaceae possui distribuição pantropical, incluindo cerca de 300 gêneros e 6.000 espécies. No Brasil ocorrem cerca de 70 gêneros e 1.000 espécies, representando uma das principais famílias da flora brasileira (SOUZA; LORENZI, 2005). Entre as espécies nacionais, encontra-se Pera glabrata (Schott) Poepp. ex Baill., que apresenta distribuição registrada nos Estados de Pernambuco, da Bahia, de Minas Gerais, do Rio de Janeiro, Espírito Santo, de São Paulo e do Paraná, sendo particularmente frequente em formações vegetais mais secas, como restinga e cerrado (PEREIRA; GOMES, 1994; ASSUMPÇÃO; NASCIMENTO, 2000; BERG; OLIVEIRA-FILHO, 2000; TALORA; MORELLATO, 2000; BOEGER; WISNIEWSKI, 2003; GOMES et al., 2004; BALDUÍNO et al., 2005; PINTO et al., 2005; SILVA et al., 2008). No cerrado do interior paulista há registro da espécie nos municípios de Brotas, Luiz Antonio, Pratânia e São Carlos (DURIGAN et al., 2002; GOMES et al., 2004; PEREIRASILVA et al., 2004; MACHADO et al., 2005; OLIVEIRA; BATALHA, 2005).

Apesar de sua ampla distribuição e de sua importância nos levantamentos fitossociológicos, até o momento não existem estudos que abordem detalhes da ecologia reprodutiva de Pera glabrata. Justifica-se, assim, a importância de estudos que elucidem os processos envolvidos na polinização e dispersão de sementes dessa espécie vegetal. O objetivo deste estudo foi verificar aspectos da ecologia reprodutiva de P. glabrata, particularmente em relação a épocas de floração e de frutificação, características morfológicas de flores, frutos e sementes, sistemas reprodutivo e de polinização e animais frugívoros responsáveis pela dispersão das sementes. Com base em observações prévias de campo, as hipóteses formuladas foram de que a espécie vegetal apresentaria polinização entomófila e dispersão ornitocórica de sementes.

\section{MATERIAL E MÉTODOS}

O estudo de campo foi desenvolvido em área de reserva legal do Campus da Universidade Federal de São Carlos (2158'S, 4753'O; 872 m alt.), onde a vegetação é de Cerrado senso strictu (COUTINHO, 1978). O solo da área é classificado como Latossolo Vermelho-Amarelo álico (LORANDI, 1985), e o clima da região pode ser classificado, segundo a sistemática de Köppen (1931), como de transição entre Cwai e Awi, isto é, clima tropical com verão úmido e inverno seco e clima quente de inverno seco.

A metodologia geral utilizada para a coleta de dados seguiu Figueiredo et al. (2008). Assim, a fenologia reprodutiva (épocas de floração e de frutificação) foi estabelecida por meio da observação quinzenal em 10 indivíduos, cinco femininos e cinco masculinos, de março de 2006 a março de 2008.

A morfologia das flores, frutos e sementes foi detalhada por análises sob microscópio estereoscópico e medições utilizando paquímetro de cinco amostras de cada uma dessas estruturas vegetais, sendo utilizada a estatística descritiva para análise dos dados (média e desvio-padrão). A emissão de odor floral perceptível ao ser humano foi verificada diretamente no campo, e a presença de néctar foi detectada visualmente após a dissecção das flores sob microscópio.

O sistema de polinização foi verificado através da observação direta de visitantes florais em indivíduos de $P$. glabrata floridos. Foram realizadas 31 h de observação, entre 6 h e 18 h, durante os meses de maio e junho de 2006 e fevereiro de 2007. O comportamento dos visitantes florais foi verificado por meio de registros visuais, e a identificação dos insetos foi feita principalmente no nível de morfoespécie, utilizando-se manuais entomológicos e comparação com coleções, da UFSCar e particulares dos autores deste artigo.

Foi testada a possibilidade de ocorrência de agamospermia, i.e., reprodução assexual, em que há formação de esporófito por meio de sementes, mas sem fusão dos gametas masculino e feminino. Nesse teste, foram escolhidos três indivíduos femininos e, em cada um deles, foram isolados botões florais de cinco ramos utilizando sacos de tecido voal, de $50 \mathrm{x}$ $50 \mathrm{~cm}$, que funcionavam como barreira aos visitantes florais. Após um mês, foi verificada a presença ou ausência de frutos e sementes.

O registro dos animais frugívoros foi feito por observação direta em indivíduos com frutos maduros, utilizando-se binóculos, e a identificação das aves foi realizada com o auxílio de guias de campo específicos para determinação em nível de espécie. Foram realizadas 18,5 h totais de observação no período diurno, entre 7 h e 18h30, no período de outubro de 2006 a fevereiro de 2007.

Revista Árvore, Viçosa-MG, v.35, n.6, p.1227-1234, 2011






\section{RESULTADOS}

Verificou-se que Pera glabrata é uma espécie dioica, com indivíduos de porte arbóreo, encontrados tanto em áreas de cerrado preservado quanto em áreas degradadas com antigo plantio de eucalipto no Campus da UFSCar.

A floração foi verificada entre os meses de fevereiro e junho, sendo encontrados frutos maduros de setembro a fevereiro. Os indivíduos masculinos e femininos florescem sincronicamente, nas mesmas épocas do ano.

Pera glabrata apresentou inflorescências cimosas, cada uma com seis a nove flores involucradas (Figura 1A). As médias e desvios-padrão do diâmetro e da altura das flores femininas foram de 4,28 mm ( $\pm 1,30$; $\mathrm{N}=5)$ e 3,96 mm ( $\pm 5,77 ; \mathrm{N}=5)$, respectivamente. Já as flores masculinas apresentaram médias de 4,28 mm ( \pm 1,48; $\mathrm{N}=5)$ do diâmetro e 5,18 mm ( $\pm 1,09 ; \mathrm{N}=5)$ da altura. Pequena fenda longitudinal ocorre nas flores em antese, tanto masculinas quanto femininas, sendo esse o único local que possibilita contato das estruturas reprodutivas com o meio externo.

Ambos os sexos apresentam flores com coloração amarelo-clara. As flores masculinas possuem anteras amareladas, pólen pulverulento, néctar na base da flor e odor levemente adocicado, perceptível de manhã, após a elevação da temperatura. Não foram detectados odor e néctar nas flores femininas, que apresentam quatro ovários de coloração esverdeada, sendo a superfície estigmática de cor amarelo-clara.

A espécie apresenta frutos simples, secos, deiscentes, com médias e desvios-padrão do diâmetro e da altura pouco antes da deiscência de $10,81 \mathrm{~mm}$ $( \pm 4,12 ; \mathrm{N}=10)$ e $11,89 \mathrm{~mm}( \pm 5,44 ; \mathrm{N}=10)$, respectivamente (Figura 1B). As sementes são ovais, pretas, com testas resistentes e envoltas parcialmente por um arilo vermelho. As médias e desvios-padrão do diâmetro, comprimento e altura das sementes foram de $3,89 \mathrm{~mm}( \pm 3,81 ; \mathrm{N}=10)$, 5,36 mm $( \pm 7,79 ; \mathrm{N}=10)$ e $2,15 \mathrm{~mm}( \pm 1,43 ; \mathrm{N}=10)$, respectivamente.

Os visitantes florais registrados foram principalmente abelhas (10 espécies), vespas (sete espécies) e moscas (12 espécies), todas de pequeno porte, além de duas espécies de besouro e uma de ave (Tabela 1). Apis mellifera e Ocyptamus sp.1 apresentaram maiores registros em visitas, com 61 e 24 observações, respectivamente.
Fonte: Fotografia cedida por R. A. de Figueiredo. Source: Photography offered by R. A. de Figueiredo.

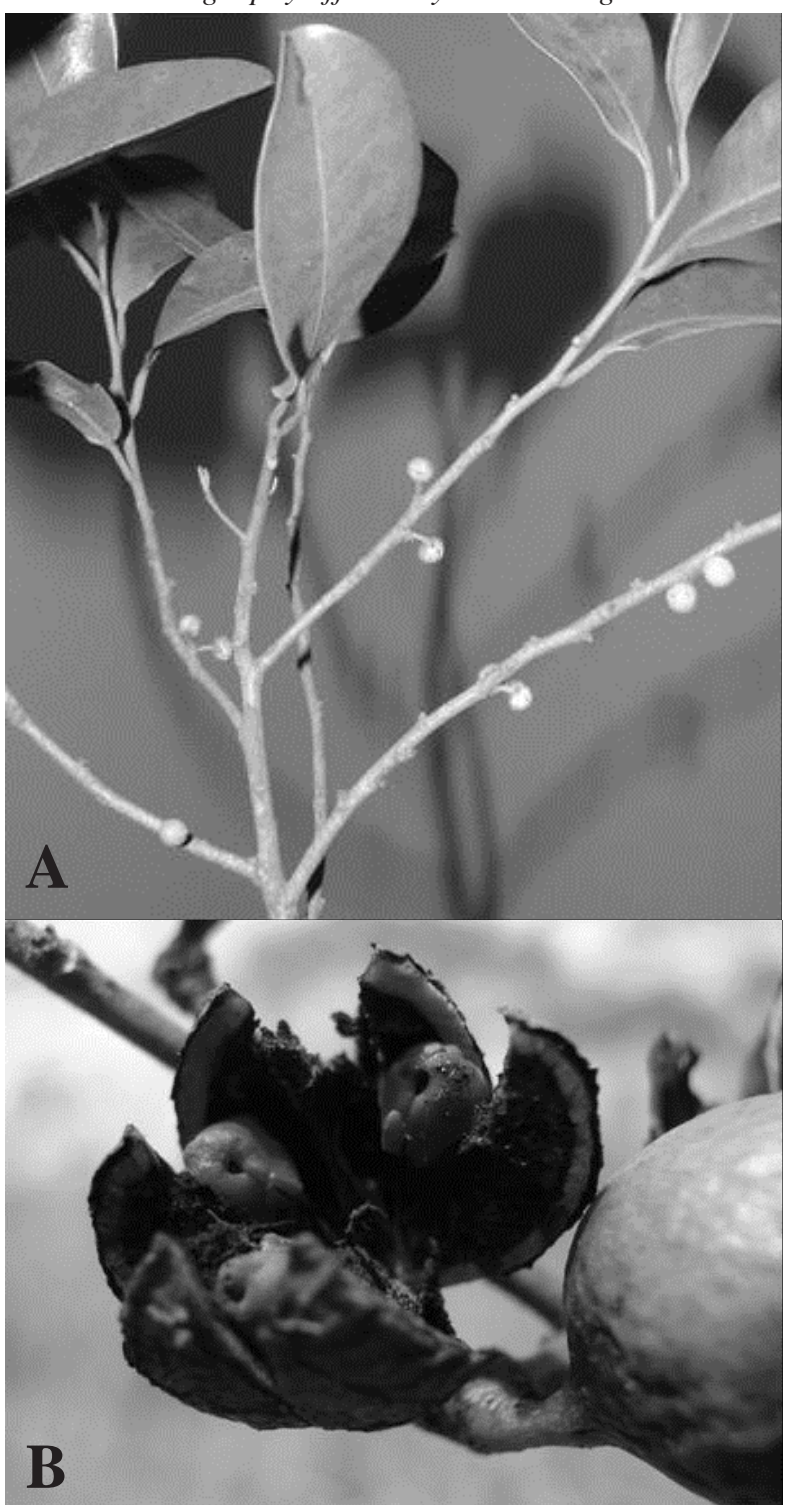

Figura 1 - Ramos com flores (A) e com fruto e sementes (B) de Pera glabrata. São Carlos, SP, Brasil.

Figure 1 - Branches with flowers (A) and with fruit and seeds (B) of Pera glabrata. São Carlos, SP, Brazil.

O comportamento geral das abelhas, vespas e moscas foi o de inserir a cabeça ou as pernas dianteiras através da fenda nas flores em antese, realizando movimentos que indicam a coleta de néctar e, ou, de pólen. A cabeça e as pernas anteriores dos insetos entram em contato direto com os órgãos sexuais das 
flores. Os insetos visitaram várias flores de cada indivíduo, demorando poucos segundos em cada uma, e algumas espécies permaneceram no indivíduo por alguns minutos. As duas espécies de coleóptero encontradas permaneceram por vários minutos dentro das flores, apresentando comportamento de predação de estruturas florais. A ave Coereba flaveola foi registrada uma única vez visitando várias flores em antese em um indivíduo, inserindo o bico através da fenda por poucos segundos e possivelmente capturando insetos.
Quatro dos cinco ramos contendo botões florais femininos ensacados produziram frutos e sementes. Os ramos não manipulados também apresentaram ampla produção de frutos e sementes. $\mathrm{O}$ indivíduo feminino no qual o ramo manipulado não produziu frutos a partir dos botões florais isolados também não produziu frutos a partir das flores não ensacadas.

Foram registradas 29 espécies de aves visitando indivíduos de P. glabrata com frutos maduros, das quais 16 foram vistas ingerindo sementes (Tabela 2).

Tabela 1 - Espécies de visitantes florais observadas em Pera glabrata. São Carlos, SP, Brasil.

Table 1 - Floral visitor species observed in Pera glabrata. São Carlos, SP, Brazil.

\begin{tabular}{|c|c|c|c|}
\hline Ordem/família & Gênero/espécie & Número de registros & Tipo de flor \\
\hline \multicolumn{4}{|l|}{ Diptera } \\
\hline \multirow[t]{11}{*}{ Syrphidae } & Copestylum sp.1 & 02 & 2 \\
\hline & Copestylum sp.2 & 02 & 2 \\
\hline & Ocyptamus sp.1 & 24 & 1,2 \\
\hline & Ocyptamus sp.2 & 04 & 1,2 \\
\hline & Ocyptamus sp.3 & 07 & 1,2 \\
\hline & Ornidia obesa & 10 & 1,2 \\
\hline & Palpada sp. & 02 & 1 \\
\hline & Salpingogaster sp.1 & 07 & 1,2 \\
\hline & Salpingogaster sp.2 & 01 & 2 \\
\hline & Syrphus sp. & 01 & 2 \\
\hline & sp. & 01 & 2 \\
\hline Família indeterminada & sp. & 02 & 2 \\
\hline \multirow[t]{2}{*}{ Coleoptera } & sp.1 & 04 & 2 \\
\hline & sp.2 & 02 & 2 \\
\hline \multicolumn{4}{|l|}{ Hymenoptera } \\
\hline \multirow[t]{2}{*}{ Andrenidae } & Oxaea flavescens & 02 & 1 \\
\hline & sp.1 & 02 & 1 \\
\hline \multirow[t]{5}{*}{ Apidae } & Apis mellifera & 61 & 1,2 \\
\hline & Trigona spinipes & 03 & 2 \\
\hline & sp.1 & 13 & 1,2 \\
\hline & sp.2 & 04 & 1 \\
\hline & sp.3 & 10 & 1 \\
\hline Colletidae & sp.1 & 02 & 2 \\
\hline \multirow[t]{2}{*}{ Halictidae } & sp.1 & 01 & 2 \\
\hline & sp.2 & 07 & 1 \\
\hline \multirow[t]{7}{*}{ Vespidae } & Brachygastra sp. & 11 & 1,2 \\
\hline & sp.1 & 12 & 1,2 \\
\hline & sp.2 & 01 & 2 \\
\hline & sp.3 & 05 & 1,2 \\
\hline & sp.4 & 02 & 1,2 \\
\hline & sp.5 & 02 & 1,2 \\
\hline & sp.6 & 01 & 2 \\
\hline \multirow[t]{3}{*}{ Família indeterminada } & sp.1 & 01 & 1 \\
\hline & sp.2 & 01 & 1 \\
\hline & sp.3 & 01 & 1 \\
\hline \multicolumn{4}{|l|}{ Passeriformes } \\
\hline Fringillidae & Coereba flaveola & 01 & \\
\hline
\end{tabular}

Tipo de flor: 1 = feminina; e 2 = masculina.

Revista Árvore, Viçosa-MG, v.35, n.6, p.1227-1234, 2011 
Tabela 2 - Aves frugívoras observadas em Pera glabrata. São Carlos, SP, Brasil. Table 2 - Frugivory birds observed in Pera glabrata. São Carlos, SP, Brazil.

\begin{tabular}{|c|c|c|}
\hline Gênero/espécie (nome popular) & Número de registros & Ingestão \\
\hline Brotogeris versiculorus (periquito-de-asa-amarela) & 01 & + \\
\hline Coereba flaveola (cambacica) & 01 & - \\
\hline Colaptes campestris (pica-pau-do-campo) & 03 & - \\
\hline Columbina minuta (rolinha-asa-de-canela) & 03 & + \\
\hline Columbina talpacoti (rolinha-caldo-de-feijão) & 05 & + \\
\hline Crotophaga ani (anu-preto) & 01 & - \\
\hline Dacnis cayana (saí-azul) & 03 & - \\
\hline Elaenia flavogaster (guaracava) & 02 & - \\
\hline Guira guira (anu-branco) & 02 & - \\
\hline Mimus saturninus (sabiá-do-campo) & 04 & - \\
\hline Myarchus sp. (maria-cavaleira) & 03 & + \\
\hline Parula pittiayumi (mariquita) & 01 & - \\
\hline Pitangus sulphuratus (bem-te-vi) & 03 & - \\
\hline Saltator similis (trinca-ferro) & 01 & - \\
\hline Tangara cayana(saíra-amarela) & 05 & + \\
\hline Thamnophilus sp. (choca) & 01 & + \\
\hline Thamnophilis puntactus (choca-bate-cabo) & 01 & + \\
\hline Thraupis sayaca (sanhaço) & 12 & + \\
\hline Turdus amaurochalinus (sabiá-роса) & 02 & + \\
\hline Turdus leucomelas (sabiá-branco)) & 06 & + \\
\hline Tyrannus melancholicus (siriri) & 01 & + \\
\hline Tyrannus savana (tesourinha) & 02 & + \\
\hline Vireo olivaceus (juruviara) & 03 & + \\
\hline Volatinia jacarina (tizil) & 01 & - \\
\hline Zonotrichia capensis (tico-tico) & 04 & + \\
\hline Não identificado 1 & 02 & + \\
\hline Não identificado 2 & 01 & + \\
\hline Não identificado 3 & 01 & - \\
\hline Não identificado 4 & 02 & - \\
\hline
\end{tabular}

+ espécies que ingeriram sementes; e - espécies que não ingeriram sementes.

Thraupis sayaca e Turdus leucomelas foram os principais frugívoros observados, com 12 e seis visitas, respectivamente.

Thraupis sayaca retira as sementes dos frutos enquanto pousa na árvore, engolindo-as inteiras, sem as macerar com o bico. Também apresentaram esse comportamento Tangara cayana e Vireo olivaceus. Já Turdus leucomelas e Turdus amaurochalinus foram registrados se alimentando tanto pousados quanto em voo. Essas espécies também engolem as sementes inteiras. Zonotrichia capensis e Colaptes campestris foram observadas ingerindo sementes caídas no chão.

\section{DISCUSSÃO}

As dimensões florais indicam que insetos de pequeno porte são os mais aptos a terem efetivo contato com as partes sexuais das flores de P. glabrata. A maioria das plantas de cerrado tem flores com morfologia que atrai ampla gama de visitantes florais (OLIVEIRA; GIBBS, 2002), especialmente as espécies dioicas (OLIVEIRA, 1996). O comportamento de forrageamento adotado pelas abelhas, vespas e moscas observadas permite que grãos de pólen sejam aderidos em suas pernas e aparelhos bucais, tornando-os potenciais polinizadores de P. glabrata, assim como verificado em outras espécies vegetais com sistema de polinização por diversos pequenos insetos (FIGUEIREDO; SAZIMA, 2004).

A agamospermia, que possibilita à espécie vegetal produzir diásporos sem a ocorrência de polinização, foi registrada em várias espécies que ocorrem em biomas secos no Brasil, sugerindo que é particularmente importante nesses ambientes (MELO et al., 1999; SALOMÃO;ALLEM, 2001;AMORIM; OLIVEIRA, 2006). 
Em espécies vegetais polinizadas por moscas ou pelo vento, a agamospermia possivelmente se desenvolve em reposta à imprevisibilidade de efetiva atuação desses agentes polinizadores (FIGUEIREDO; SAZIMA, 2007).

As flores femininas de $P$. glabrata possivelmente têm na coloração a sua única forma de atração de visitantes florais, por não liberarem odor e não oferecerem recursos alimentares perceptíveis. Se assim for, ocorre a polinização por engodo ("deceipt pollination”), uma vez que a similaridade entre as flores masculinas e femininas deve induzir indistintamente a visitação por insetos. A polinização por engodo ocorre em espécies que apresentam flores femininas morfologicamente similares às flores masculinas ou quando possuem um par mimético no local de ocorrência, tendo essa forma de polinização sido registrada em espécies de Clusiaceae (CARMO; FRANCESCHINELLI, 2002), Cucurbitaceae (LENZI et al., 2005) e Orchidaceae (ALMEIDA; FIGUEIREDO, 2003; BORBA; BRAGA, 2003).

A floração massiva tanto nos indivíduos femininos quanto nos masculinos possivelmente pode agir como fator atrativo a visitantes florais oportunistas, como pequenas moscas, abelhas e vespas (CROAT, 1979).

Pera glabrata apresentou-se ornitocórica quanto ao modo de dispersão de diásporos. Os frutos bem expostos e a coloração vistosa das sementes ariladas certamente contribuem para a atração das aves. Francisco et al. (2007) haviam registrado 25 espécies de aves se alimentando de sementes de $P$. glabrata. Como a maior parte das aves não apresentou o comportamento de maceração da semente antes da deglutição, poucas sementes caíam sob a copa da planta-mãe, o que, segundo alguns autores (JANZEN, 1970; PEDRONI, 1995), indica que a espécie tem aumentadas suas chances de sobrevivência e potencial para a colonização de novas áreas.

Alguns estudos têm sugerido relação entre a presença de $P$. glabrata e áreas impactadas, particularmente devido ao seu alto Índice de Cobertura Vegetal - IVC, como verificado em áreas de restinga com formações arbustivas e arbóreas (MONTEIRO; CÉSAR, 1995; ASSUMPÇÃO; NASCIMENTO, 2000). A área onde se realizou este estudo também pode ser considerada impactada, pois foi afetada por diversos incêndios e plantios de eucalipto ao longo do tempo. Por ser espécie que se instala com facilidade em regiões sob o impacto ou em processo de regeneração e que exerce atração sobre uma diversidade considerável de insetos e aves, $P$. glabrata pode ser considerada espécie de relevância nos planos de conservação e recuperação de áreas degradadas.

\section{REFERÊNCIAS}

ALMEIDA, A. M.; FIGUEIREDO, R. A. Ants visit nectaries of Epidendrum denticulatum (Orchidaceae) in a Brazilian rainforest: effects on herbivory and pollination. Brazilian Journal of Biology, v.63, n.4, p.551-558, 2003.

AMORIM, F. W.; OLIVEIRA, P. E. Estrutura sexual e ecologia reprodutiva de Amaioua guianensis Aubl. (Rubiaceae), uma espécie dióica de formações florestais de cerrado.

Revista Brasileira de Botânica, v.29, n.3, p.353-362, 2006.

ASSUMPÇÃO, J.; NASCIMENTO, M. T. Estrutura e composição florística de quatro formações vegetais de restinga no complexo lagunar Grussaí/Iquipari, São João da Barra, RJ, Brasil. Acta Botanica Brasilica, v.14, n.3, p.301-315, 2000.

BALDUINO, A. P. C. et al. Fitossociologia e análise comparativa da composição florística no cerrado da flora de Paraopeba - MG. Revista Árvore, v.29, n.1, p.25-34, 2005.

BERG, E. V. D.; OLIVEIRA-FILHO, A. T. Composição florística e estrutura fitossociológica de uma floresta ripária em Itutinga, MG, e comparação com outras áreas. Revista Brasileira de Botânica, v.23, n.3, p.231-253, 2000.

BOEGER, M. R. T.; WISNIEWSKI, C. Comparação da morfologia foliar de espécies arbóreas de três estádios sucessionais distintos de floresta ombrófila densa (Floresta Atlântica) no Sul do Brasil. Revista Brasileira de Botânica, v.26, n.1, p.61-72, 2003.

BORBA, E. L.; BRAGA, P. I. S. Reproductive biology of Pseudolaelia corcovadensis Porto \& Brade (Orchidaceae): melittophyly and self-compatibility in a basal Laeliinae. Revista Brasileira de Botânica, v.26, n.4, p.541-549, 2003. 
CARMO, R. M.; FRANCESCHINELLI, E. V. Polinização e biologia floral de Clusia arrudae Planchon \& Triana (Clusiaceae) na Serra da Calçada, município de Brumadinho, MG. Revista Brasileira de Botânica, v.25, n.3, p.351-360, 2002.

COUTINHO, L. M. O. Conceito de cerrado. Revista Brasileira de Botânica, v.1, n.1, p.17-23, 1978.

CROAT, T. B. The sexuality of Barro Colorado Island flora (Panama). Phytologia, v.42, n.4, p. 319-348, 1979.

DURIGAN, G. et al. Caracterização de dois estratos da vegetação em uma área de Cerrado no município de Brotas, SP, Brasil. Acta Botanica Brasilica, v.16, n.3, p.251-262, 2002.

FIGUEIREDO, R. A.; SAZIMA, M. Pollination ecology and resource partitioning in neotropical pipers In: DYER, L.; PALMER, A. (Eds.) Piper, a model genus for studies of phytochemistry, ecology, and evolution. New York: Kluwer/Plenum Publishers, 2004. p.33-57.

FIGUEIREDO, R. A.; SAZIMA, M. Phenology and pollination biology of eight Peperomia species (Piperaceae) in semideciduous forests in Southeastern Brazil. Plant Biology, v.9, n.1, p.136-141, 2007.

FIGUEIREDO, R. A. et al. Reproductive ecology of the exotic tree Muntingia calabura L.

(Muntingiaceae) in southeastern Brazil. Revista Árvore, v.32, n.6, p.993-999, 2008.

FRANCISCO, M. R.; LUNARDI, V. O.; GALETTI, M. Bird attributes, plant characteristics, and seed dispersal of Pera glabrata (Schott, 1858),

(Euphorbiaceae) in a disturbed cerrado area.

Revista Brasileira de Biologia, v.67, n.4, p.627-634, 2007.

GOMES, B. Z.; MARTINS, F. R.; TAMASHIRO, J. Y. Estrutura do cerradão e da transição entre cerradão e floresta paludícola num fragmento da International Paper do Brasil Ltda., em Brotas, SP. Revista Brasileira de Botânica, v.27, n.2, p.249-262, 2004.
JANZEN, D. H. Herbivores and the number of tree species in tropical forests. American Naturalist, v.104, n.940, p.501-528, 1970.

KÖPPEN, W. Climatología. México: Fondo de Cultura Económica, 1931.

LENZI, M.; ORTH, A. I.; GUERRA, T. M. Pollination ecology of Momordica charantia L. (Cucurbitaceae) in Florianópolis, SC, Brazil.

Revista Brasileira de Botânica, v.28, n.3, p.505-513, 2005.

LORANDI, R. Caracterização dos solos das áreas urbana e suburbana de São Carlos (SP) e suas aplicações. 1985. 151 f. Tese (Doutorado em Solos e Nutrição de Plantas) - Escola Superior de Agronomia Luiz de Queiróz, Piracicaba, 1985.

MACHADO, S. R.; BARBOSA, S. B.; CAMPOS, J. C. Cerrado Palmeira da Serra: guia de campo ilustrado. São Carlos: Rima, 2005.

MELO, G. F.; MACHADO, I. C.; LUCENO, M. Reprodución de três especies de Clidemia (Melastomataceae) en Brasil. Revista de Biología Tropical, v.47, n.3, p.359-363, 1999.

MONTEIRO, R.; CÉSAR, O. Padrões espaciais em espécies arbóreas na planície litorânea de Picinguaba, Parque Estadual da Serra do Mar, Ubatuba - SP. Arquivos de Biologia e Tecnologia, v.38, n.2, p.533-540, 1995.

OLIVEIRA, F. F.; BATALHA, M. A. Lognormal abundance distribution of woody species in a cerrado fragment (São Carlos, southeastern Brazil). Revista Brasileira de Botânica, v.28, n.1, p.39-45, 2005.

OLIVEIRA, P. E. Dioecy in the cerrado vegetation of Central Brasil. Flora, v.191, p.235-243, 1996.

OLIVEIRA, P. E.; GIBBS, P. E. Pollination and reproductive biology in cerradão plant communities. In: OLIVEIRA, P. E.; MARQUIS, R. J. (Eds.) The cerrados of Brazil: ecology and natural history of a Neotropical savanna. New York: Columbia University Press, 2002. p.329-347.

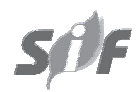

Revista Árvore, Viçosa-MG, v.35, n.6, p.1227-1234, 2011 
PEDRONI, F. A ecologia da copaíba. In:

MORELLATO, L. P. C.; LEITÃO-FILHO, H. F.

(Eds.) Ecologia e preservação de uma

floresta tropical urbana: Reserva de Santa

Genebra. Campinas: Unicamp, 1995. p.70-76.

PEREIRA, O. J.; GOMES, J. M. L. Levantamento florístico das comunidades vegetais de restinga no município de Conceição da Barra-ES. In:

SIMPÓSIO DE ECOSSISTEMAS DA

COSTA BRASILEIRA, ACIESP, 3., São

Paulo, 1994. Anais... São Paulo, 1994. p.67-78.

PEREIRA-SILVA, E. F. L. et al. Florística e fitossociologia dos estratos arbustivo e arbóreo de um remanescente de cerradão em uma unidade de conservação do Estado de São Paulo. Revista Brasileira de Botânica, v.27, n.3, p.533-544, 2004.

PINTO, L. V. A. et al. Distribuição das espécies arbóreas-arbustivas ao longo do gradiente de umidade do solo de nascentes pontuais da bacia hidrográfica do Riberião Santa Cruz, Lavras, MG. Cerne, v.11, n.3, p.294-305, 2005.
SALOMÃO, A. N.; ALLEM, A. C.

Polyembryony in angiospermous trees of the Brazilian cerrado and caatinga vegetation.

Acta Botanica Brasilica, v.15, n.3, p.369-378, 2001.

SILVA, S. L. L.; ZICKEL, C. S.; CESTARO, L. A. Flora vascular e perfil fisionômico de uma restinga no litoral sul de Pernambuco. Acta Botanica Brasilica, v.22, n.4, p.1123-1135, 2008.

SOUZA, V. C.; LORENZI, H. Botânica sistemática: guia ilustrado para identificação das famílias de Angiospermas da flora brasileira, baseado em APG II. Nova Odessa: Instituto Plantarum, 2005.

TALORA, D. C.; MORELLATO, L. P. C. Fenologia de espécies arbóreas em floresta de planícies litorâneas no sudeste do Brasil.

Revista Brasileira de Botânica, v.23, n.1, p.13-26, 2000. 